\title{
Sh 2-68 - A Planetary Nebula Leaving it's Mark on the Interstellar Medium
}

\author{
F. Kerber
}

ST-ECF, Karl-Schwarzschild-Str.2, D-85748 Garching, Germany

F. Guglielmetti

STScI, 3700 San Martin Drive, Baltimore, MD 21218, USA

R. Mignani

ESO, Karl-Schwarzschild-Str.2, D-85748 Garching, Germany

M. Roth

Las Campanas Observatory, Carnegie Institution of Washington, Casilla 601, La Serena, Chile

\begin{abstract}
Using $\mathrm{H} \alpha$ images from the Southern H-Alpha Sky Survey Atlas (SHASSA) we have discovered a "cometary tail" of ionized matter extending at least 30 arcmin from the main body of the planetary nebula Sh 2-68 (PN G030.6+06.2). This tail is aligned with the proper motion of the central star and is obviously a contrail of material left behind by Sh 2-68 moving in a Galactic Disk orbit. The tail's existence is a direct confirmation of Sh 2-68's interaction with the interstellar medium (ISM) and highlights the possibly important role of the ISM's magnetic field.
\end{abstract}

\section{Introduction}

Over recent years, interaction of planetary nebulae (PNe) with the surrounding ISM has been recognized as a very important process in the late stages of PN evolution, see Kerber et al. (2002) for references. The interaction impedes and finally stops the expansion of the nebula; then the Galactic motion of the central star (CS) makes it leave the center of the PN. Due to the recent discovery of the proper motion (PM) of its CS (Kerber et al., 2002) Sh 2-68 is now a prime example for PN-ISM interaction and the return of processed matter to the ISM.

\section{Observation and Discussion}

Using SHASSA (Gaustad et al. 2001) we discovered a tail emanating from Sh 268. Fig. 1 actually is a co-addition of two median-filtered continuum-subtracted frames. The original resolution is $0.8^{\prime} / \mathrm{pixel}$. The tail extends for at least $30^{\prime}$ beyond the previously known size of $14^{\prime}$ (Xilouris et al., 1996) giving the PN a total length of more than $45^{\prime}$. The tail has a position angle (PA) of $212 \pm 10^{\circ}$, 


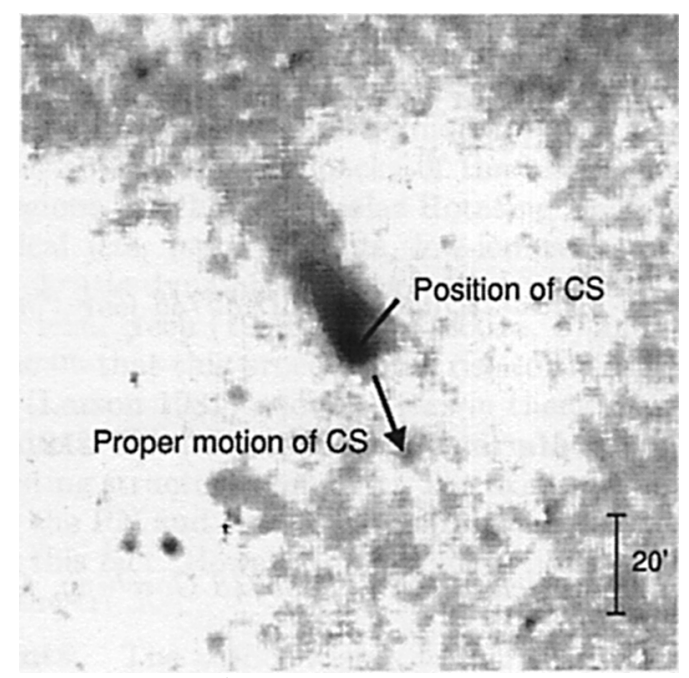

Figure 1. $\quad \mathrm{H} \alpha$ image of Sh 2-68 from SHASSA (logarithmic scale).

which is fully consistent with that of the central star's PM of $202 \pm 6^{\circ}$ (Kerber et al., 2002). At the spatial resolution of the SHASSA little structure can be discerned in the contrail. Still, this is currently our best view of a PN returning processed matter to the ISM.

For the measured PM of $53.2 \mathrm{mas} / \mathrm{yr}$, the CS was located at the far end of the tail 45,000 years ago, proving that an ionized nebula existed even then. This is a lower limit on the age, as the tail may extend further than currently seen and, when the matter was stripped, it retained a significant fraction of the star's PM and fell behind the nebula only gradually. Moreover, recombination becomes important on the above time scales. Soker \& Dgani (1997) showed that such stripping of nebular material is likely to occur over a wide range of PN/ISM parameters for PNe located close to the Galactic plane and in the presence of a significant magnetic field. Once we have determined the distance of Sh 2-68, it will be possible to quantitatively test the theory of PN-ISM interaction.

Acknowledgments. We acknowledge the use of the Southern H-Alpha Sky Survey Atlas (SHASSA), which is supported by the National Science Foundation. This research has made use of the SIMBAD database, operated at CDS, Strasbourg, France.

\section{References}

Gaustad, J.E., McCullough, P.R., Rosing, W., \& Van Buren, D. 2001, PASP, 113, 1326 Kerber, F. Guglielmetti, F., Mignani, R., \& Roth, M. 2002, A\&A 381, L 9

Soker, N., \& Dgani, R. 1997, ApJ, 484, 277

Xilouris, K. M., Papamastorakis, J., Paleologou, E., \& Terzian, Y. 1996, A\&A, 310, 603 